\title{
DARK MATTER IN THE UNIVERSE
}

\author{
A.D. Dolgov \\ TAC, Juliane Maries Vej 30, DK-2100, Copenhagen, Denmark \\ Also: ITEP, Bol. Cheremushkinskaya 25, Moscow 113259, Russia \\ E-mail:dolgov@tac.dk
}

\begin{abstract}
Cosmological arguments proving that the universe is dominated by invisible nonbaryonic matter are reviewed. Possible physical candidates for dark matter particles are discussed. A particular attention is paid to non-compensated remnants of vacuum energy, to the question of stability of super-heavy relics, cosmological mass bounds for very heavy neutral lepton, and some other more exotic possibilities.
\end{abstract}

\section{Introduction}

Probably one of the most important discoveries of this Century was the discovery that the universe consists mostly of an unknown form of matter. This matter neither emit nor absorb light and got the name dark (or better to say, invisible) matter. It is observed only indirectly through its gravitational action and, though there are plenty of theoretical hypotheses, the nature of dark matter remains mysterious. First hints on existence of dark matter were found more than half of a century ago [1, 2]. Velocity dispersion of astronomical objects was larger than one would expect from observation of luminous matter. The fact that there is more mass than light in the universe, got a strong support only 40 years later. It was initiated by two groups [3, [4 and stimulated a burst of activity in the field. Now there are a large amount of accumulated astronomical data that unambiguously prove that the universe is dominated by an invisible matter or to be more precise there is much more gravity in the universe than all the visible matter could provide.

Very strong arguments in favor of invisible cosmic matter follow from the so called galactic rotational curves, i.e. from the observed dependence of velocities of gravitationally bound bodies on the distance from the visible center. A very well known example of rotational curves that have led to the seminal discovery of the Newton gravitational law is the distribution of velocities of planets in the Solar system (see fig. 1, taken from ref. [5]).

On the basis of this data it was concluded that gravitational forces drops down with distance as $F \sim 1 / r^{2}$ and correspondingly, by the virial theorem, $v^{2}(r) \sim G_{N} M(r) / r$, so that $v \sim 1 / \sqrt{r}$ for point-like central mass; here $M(r)$ is the mass of gravitating matter inside the radius $r$. However measurements of rotational velocities of gas around galaxies produce a very different picture, $v(r)$ does not go down to zero with an increasing distance from the luminous center but tends to a constant value, see fig. 2 [6]. At the present day more than 1000 galactic rotational curves are measured (see e.g. [7]) and they show a similar behavior. It is quite a striking fact that rotational curves are very accurately flat at large distances, $v \rightarrow$ const. If such curves were observed at Keoler-Newton's time one might conclude that the oravitational force did not 


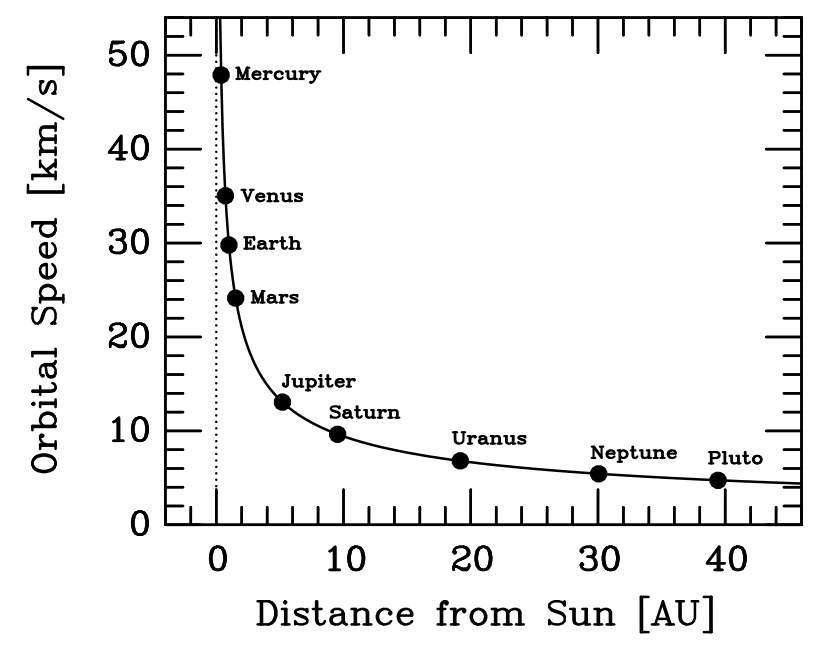

Figure 1: Rotation curve of the solar system which falls off as $1 / \sqrt{r}$ in accordance with Kepler's law. The astronomical unit (AU) is the Earth-Sun distance of $1.50 \times 10^{13} \mathrm{~cm}$.

obey the famous inverse square law but something quite different, $F \sim 1 / r$, with the potential $U \sim \ln r$. However it is very difficult, if possible at all, to modify beautiful general relativity at large distances in such a way that it would give $1 / r$-forces. A normal interpretation of flat rotational curves is that there is an invisible matter around galaxies with mass density decreasing as

$$
\rho \sim 1 / r^{2}
$$

and correspondingly $M(r) \sim r$. Such mass distribution could be in a self-gravitating isothermal gas sphere. However, if the dark matter particles do not possess a sufficiently strong selfinteraction it is not clear how they would acquire thermal equilibrium.

It is not yet established how far the law (11) remains valid. If it is true up to neighboring galaxies, the average mass density of this invisible matter would be rather close to the critical one

$$
\rho_{c}=\frac{3 H^{2}}{8 \pi G_{N}} \approx 1.86 \cdot 10^{-29} h_{100} \mathrm{~g} / \mathrm{cm}^{3}
$$

where $h_{100}=H / 100 \mathrm{~km} / \mathrm{s} / \mathrm{Mpc}$ is dimensionless Hubble constant; by the most recent data [8] $h_{100} \approx 0.7$ with the error bars of about $10-15 \%$; for a review see ref. 9].

The contributions of different forms of matter to the cosmological mass/energy density according to the present day data is the following. The visible luminous matter contributes very little to total density [10]:

$$
\Omega_{\text {lum }}=\rho_{\text {lum }} / \rho_{c} \leq 0.003 h_{100}^{-1}
$$

There could be much more non-luminous baryons in the forms of faint stars, gas, etc (see below sec. 2) but the standard theory of primordial nucleosynthesis does not allow too high mass fraction of baryonic matter. It is probably a proper time and place to mention that George Gamow [1] made a pioneering contribution to big bang nucleosynthesis. Abundances of light elements are sensitive to total number fraction of cosmic baryons, more precisely abundances of light elements depend upon the ratio of number densities of baryons to photons, $\eta_{10}=$ $10^{10} n_{h} / n_{1}$ Comparing theoretical predictions with observations one can deduce the value of 

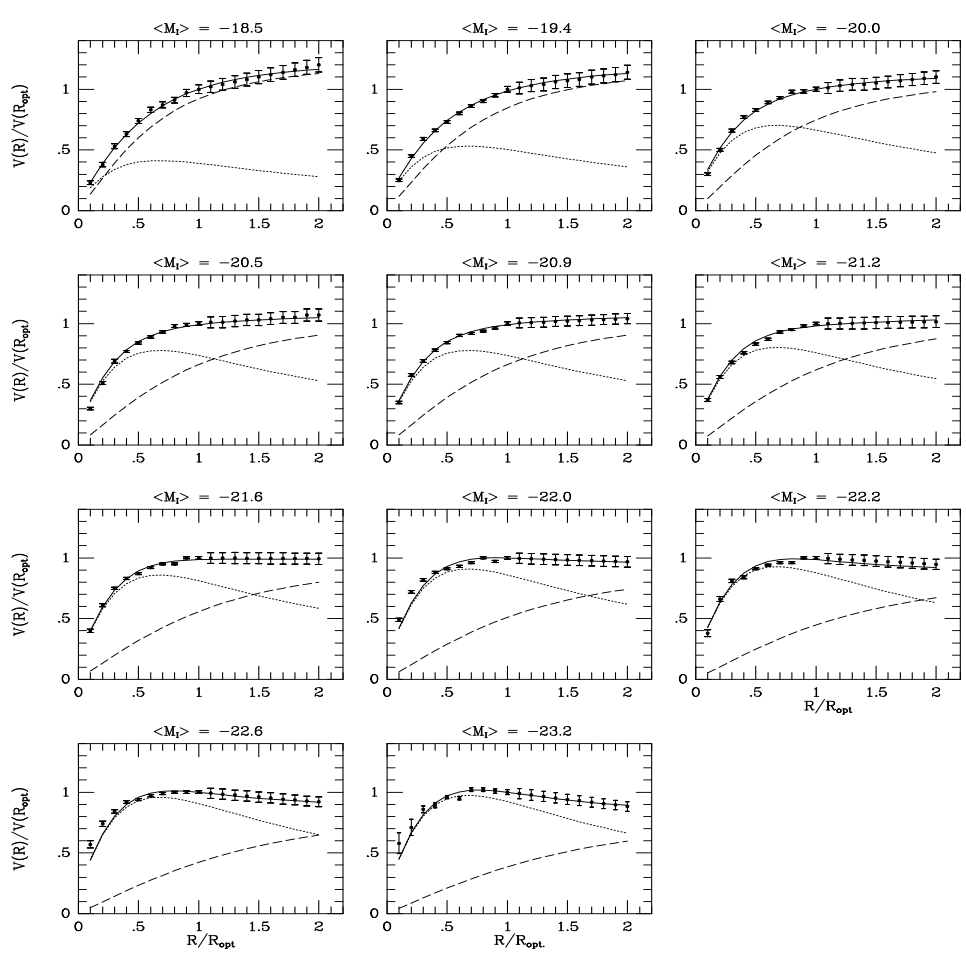

Figure 2: Coadded rotation curves (filled circles with error bars) reproduced by universal rotational curve (solid line) Also shown the separate dark/luminous contributions (dotted line: disk; dashed line: halo.)

this ratio at nucleosynthesis. The result crucially depends upon the observed abundance of deuterium since the latter is especially sensitive to $\eta$. There are two conflicting pieces of data: high and low deuterium, see discussion and references in the review [12]. For low ${ }^{2} H$ regions the limits presented in ref. [12] are:

$$
\Omega h_{100}^{2}=0.015-0.023 \text { and } \eta_{10}=4.2-6.3
$$

while for high ${ }^{2} H$ :

$$
\Omega h_{100}^{2}=0.004-0.01 \text { and } \eta_{10}=1.2-2.8
$$

Most probably one or other of the above is incorrect and the predominant attitude is in favor of low deuterium. However, it would be extremely interesting if both are true, so that the abundance of primordial deuterium is different in different regions of the universe. A possible explanation of this phenomenon is a large and spatially varying neutrino degeneracy that predicts a large mass fraction of primordial helium, more than $50 \%$, comparing to $\sim 25 \%$ in normal deuterium regions (that were called "low" above), and quite low helium, $\leq 12 \%$, in the anomalously low deuterium regions [13].

Anyhow, independently of these subtleties, big bang nucleosynthesis strongly indicates that the mass fraction of normal baryonic matter in the universe is quite small (see also the discussion below in sec. 2). On the other hand, the amount of gravitating matter, found by different dynamical methods (for a review see [14]), gives $\Omega_{m} \sim 0.3$. These methods are sensitive to clustered matter and do not feel uniformly distributed energy/mass density. Theoretical prediction based on inflationary model is $\Omega_{t o t}=1 \pm 10^{-4}$. This number may be compatible with the above quoted value for $\Omega_{m}$ only if the rest of matter is uniformly distributed. The recent indications to a non-zero cosmological constant [15] with 
permit to fill the gap between 0.3 and 1 . It is possibly too early to make a definite conclusion, since the result is very important and all possible checks should be done. Moreover the SNdata that led to the conclusion of non-zero $\Lambda$ might be subject to a serious criticism [16]. Still the combined different astronomical data quite strongly suggest that cosmological constant is indeed non-zero.

The attitude to a possibly non-vanishing cosmological constant from different prominent cosmologists and astrophysicists were and is quite diverse. For example Einstein, who "invented" cosmological constant and introduced it into general relativity, considered it as the biggest blunder of his life. The attitude of Gamow was similar, he wrote in his autobiography book [17]: " $\lambda$ again rises its nasty head" On the other hand, Lemaitre and Eddington considered $\Lambda$ very favorably. Moreover, a non-zero $\Lambda$ (or what is the same, vacuum energy) should be quite naturally non-zero from a particle physicist's point of view, though any theoretical estimate by far exceeds astronomical upper limits (see discussion in sec. 4).

To conclude, it seems very probable that the normal baryonic matter contributes only a minor fraction to the total mass/energy of the universe and we will discuss below possible forms of this yet unknown but dominant part of our world. It is not excluded that there is not a single form of dark matter. The data request several different ones and if it is indeed the case the mystery becomes even deeper. In particular, one has to understand the so called cosmic conspiracy: why different forms of dark matter give comparable contributions to $\Omega$, while they naturally would differ by many orders of magnitude.

\section{Baryonic dark matter.}

Since an idea that there is a cosmic ocean of an absolutely unknown matter is quite drastic, one is inclined to look for less revolutionary explanations of the data. The first natural question is if all the dark matter, possibly excluding vacuum energy, could be the normal baryonic staff somehow hidden from observation. The relevant discussion of the cosmic baryon budget can be found in ref. 18

As we have already mentioned in the Introduction a very strong upper limit on the total amount of baryons in the universe follows from the Big Bang Nucleosynthesis. However this limit would be invalid if for example electronic neutrinos are strongly degenerate [19, 20]. A charge asymmetry in electronic neutrinos corresponding to dimensionless chemical potential $\mu_{\nu_{e}} / T \sim 1$ could significantly loosen the bound on baryonic mass density and make it close to the necessary $0.3 \rho_{c}$.

However there are some other data that make it very difficult to have baryon dominated universe. Strong arguments against this possibility come from the theory of large scale structure formation. In the case of adiabatic perturbations that are characterized by approximate equality of density and temperature fluctuations, $\delta \rho / \rho \sim \delta T / T$, there is too little time for cosmic structures to evolve. Indeed the perturbations in the baryonic matter could rise only after hydrogen recombination that took place rather late at redshift $z \approx 10^{3}$. After that the perturbations might rise only as the scale factor so to the present time they at most could be amplified by this factor of $10^{3}$. However, it is well known that the fluctuations of the CMB (cosmic microwave background) temperature are quite small, $\delta T / T<$ a few $\times 10^{-5}$. Hence even today the density fluctuations should be quite small in contrast to the observed developed structures with $\delta \rho / \rho \gg 1$.

For isocurvature perturbations the fluctuations of CMB temperature are much smaller than density perturbations, $\delta T / T \ll \delta \rho / \rho$, and this permits to avoid the above objection. However 
if it were the case, the spectrum of angular fluctuations of CMB would be quite different from the observed one. In particular, the first acoustic peak would be near $l=400$, while the data strongly indicates that this peak is close to $l=200$ in agreement with adiabatic theory (for a recent review and the list of references see e.g. ref. [21]). This argument can be avoided if the shift of the acoustic peak to higher $l$ is compensated by the curvature effects (I thank J. Silk for indication to this point).

Another weighty argument against baryonic universe is that it is practically impossible to conceal $90 \%$ of baryons. Baryonic matter strongly interacts with light and even if the baryons are nonluminous themselves, they would strongly absorb light. So baryonic matter should be observed either in emission or absorption lines. There is not much space for baryons to escape detection:

1. Cold gas or dust do not emit light but can be observed by absorption lines (Gunn-Peterson test).

2. Hot gas is seen by X-rays if it is clumped, if it is diffuse it would distort CMB spectrum.

3. Neutron stars or "normal" black holes" that were produced as a result of stellar evolution, would contaminate interstellar medium by "metals" (elements that are heavier than ${ }^{4} \mathrm{He}$ ).

4. Dust is seen in infrared

According to ref. 18 the total baryon budget is in the range:

$$
0.007 \leq \Omega_{B} \leq 0.041
$$

with the best guess $\Omega_{B}=0.021$ (for $h_{100}=0.7$ ).

A special search was performed for the so called $M A C H O$ 's (massive astrophysical compact halo objects). They may include brown dwarfs, low luminosity stars, primordial black holes. Such objects are not directly visible and they were looked for through gravitational microlensing [22]. The search was pioneered by MACHO [23] and EROS [24] collaborations and at the present time about a hundred of such objects were found in the Galaxy and in the nearby halo. According to the EROS results the mass density of the micro-lenses with the masses in the interval $\left(5 \cdot 10^{-8}-10^{-2}\right) M_{\odot}$ is less than $0.2 \rho_{\text {Halo }}$. The MACHO observations permit to make the conclusion that the masses of micro-lensing objects lies in the interval $(0.1-1.0) M_{\odot}$ at $90 \% \mathrm{CL}$. The mean value of the mass is about $0.5 M_{\odot}$.

Instead of approaching to the resolution of the problem of dark matter, these observations made things even more mysterious and more interesting. A large mass of MACHO's suggests that they could be the remnants of the usual stars (white dwarfs?). However it is difficult to explain their relatively large number density and distribution. They could be primordial black holes but in this case they are not necessarily baryonic. An intriguing possibility is that they are the so called mirror or shadow stars, i.e they are formed from a new form of matter that is related to ours only gravitationally and possibly by a new very weak interaction (see sec. 8).

Anyhow, baryons seem to contribute only a minor fraction to the total mass of the universe and some new form of matter should exist. There is no shortage of possible candidates but it remains unknown what one (or maybe ones) is (are) the real dominating entity. 


\section{Non-baryonic (exotic?) dark matter; what is it?}

For an astronomer the classification of dark matter from the point of view of large scale structure formation is especially relevant. Independently of its physical nature cosmological dark matter can be of the following three types:

1. Hot dark matter (HDM). For this form of dark matter the structure can be originally formed only at very large scales, much larger than galactic size, $l_{\text {str }} \gg l_{\text {gal }}$.

2. Cold dark matter (CDM). It is an opposite limiting case for which the structure is formed at the low scale, $l_{s t r} \ll l_{\text {gal }}$.

3. Warm dark matter (WDM). This is an intermediate case when the characteristic scale of the structures is of the order of galactic size, $l_{\text {str }} \sim l_{\text {gal }}$.

Somewhat separately there stands $\Lambda$-term or, what is the same, vacuum energy. There are some rather strong indications that for a good description of the observed large scale structure several different forms of dark matter, including $\Lambda$-term, may be necessary.

Another astronomically important feature of dark matter is its dissipation properties. If dark matter easily loose energy, the structure formation could proceed faster. In the opposite case the cooling of dark matter would be less efficient and the structures on small scales would not be formed. So from this point of view there could be two forms of dark matter, dissipationless and/or dissipative. The dominant part of physical candidates for dark matter particles are weakly interacting and thus dissipationless. However there are some, possibly more exotic, models supplying strongly interacting dark matter particles that could easily loose energy.

There are quite many physically possible, and sometimes even natural, candidates for dark matter particles. An abridged list of them in the order indicating the author's preference is the following:

1. Massive neutrinos.

2. Non-compensated remnant of vacuum energy.

3. New not yet discovered, but theoretically predicted, elementary particles: lightest supersymmetric particle, axion, majoron, unstable but long-lived particles, super-heavy relics, etc. It is even possible to construct models in which the same kind particles would contribute e.g. both to hot and warm dark matter.

4. New shadow or mirror world.

5. Primordial black holes.

6. Topological defects (topological solitons).

7. Non-topological solitons.

8. Neither of the above.

It is quite possible that the last entry at the bottom of the list may happen after all to become the first. 


\section{Vacuum energy}

The problem of vacuum energy is possibly the most striking in the contemporary physics. Any reasonable theoretical estimate disagree with the astronomical upper limits on $\rho_{v a c}$ by 50-100 orders of magnitude (for a review see refs. [25, 26]). In fact there are practically experimentally proven contributions into vacuum energy from the known in quantum chromodynamics (QCD) vacuum condensates of quarks and gluons. The existence of these condensates is necessary for correct description of hadron properties. In this sense the existence of these condensates is an experimental fact. So we have a fantastic situation: there are well established contributions into vacuum energy that are larger than the permitted value by the factor $10^{47}$. It may only mean that there is some extremely accurate mechanism that compensates this huge amount practically down to zero. Here "zero" is in the scale of elementary particle physics; on astronomical scale the remaining vacuum energy may be quite significant. This compensation should be achieved by something that is not directly related to quarks and gluons because all the light fields possessing QCD interactions are known, while heavy fields cannot make a compensation with the desired accuracy.

It is tempting to assume that the curvature of space-time created by vacuum energy would generate a vacuum condensate of a new massless (or extremely light) field $\Phi$ and the energy of the condensate would cancel down the original vacuum energy in accordance with the famous Le Chatelier's principle. It is closely analogous to the axionic mechanism of natural CPconservation in QCD. Generic features that one should expect from such compensating (or adjustment) mechanism are quite interesting. First, the compensation is never complete, the amount of non-compensated vacuum energy is always parametrically of the order of critical energy:

$$
\Delta \rho_{v a c}=\rho_{v a c}^{i n}-\rho_{\Phi} \sim\left(m_{P l}^{2} / t^{2}\right),
$$

but the coefficient of proportionality may be different at different stages of the evolution of the universe (e.g. at MD- and RD-stages). Another unusual feature is that the equation of state of the dark matter corresponding to $\Delta \rho_{\text {vac }}$ may be very much different from the standard ones, $p=\rho / 3$ at RD-stage or $p=0$ at MD-stage.

So hopefully such compensating mechanism may be able not only to cut the "nasty head of $\lambda$ " (using Gamow words) but also to extinguish it almost down to nothing with only a small tail remaining. In fact, it is exactly this small tail that induced such a strong negative reaction from Gamow, because it could be $100 \%$ cosmologically relevant. This demonstrates two sides of the cosmological constant problem. Astronomers put the question if it is cosmologically important, i.e. if $\rho_{\text {vac }}$ is not negligible in comparison with $\rho_{c}$. If the answer is affirmative, then another puzzling problem appears: why vacuum energy, that remains constant in the course of the cosmological expansion, is close today to $\rho_{c}$ which evolves as $1 / t^{2}$ ? Particle physicists are more puzzled by the question why vacuum energy does not exceed $\rho_{c}$ by almost an infinite amount. However if this is somehow arranged, then the natural value should be precisely zero. So astronomical indications that $\rho_{v a c}$ may be non-vanishing are of prime importance for all members of astro-particle community.

The compensation mechanism would successfully address both issues: it permits to compensate $\rho_{\text {vac }}$ to cosmologically acceptable value and gives a non-compensated remnant of the

order of $\rho_{c}$ at any period of the history of the universe. However all that are predictions of a non-existing theory. Original compensating mechanism [27] is based on a massless scalar field with the Lagrangian: 
where $R$ is the curvature scalar. For a certain choice of the sign of the constant $\xi$ the field $\Phi$ becomes unstable in De Sitter background (the term $\xi R^{2}$ behaves as a negative mass squared) and a vacuum condensate of $\Phi$ would evolve. The back-reaction of this condensate on the expansion results in a change from the exponential De Sitter regime to a more slow Friedman one, $a(t) \sim t^{\alpha}$. So far so good, but this change of the regime was not achieved by the compensation of the vacuum energy. In fact the energy-momentum tensor of $\Phi$ does not have the vacuum form, it is not proportional to the metric tensor $g_{\mu \nu}$. The slowing of the expansion is achieved by the decrease of the gravitational coupling constant with time, $G_{N} \sim 1 / t^{2}$.

Other possible candidates on the role of the compensating field could be fields with higher spins, vector or tensor ones [28]. More promising seems to be symmetric tensor field $\Phi_{\mu \nu}$. Even the simplest possible Lagrangian:

$$
\mathcal{L}_{2}=\Phi_{\mu \nu ; \alpha} \Phi^{\mu \nu ; \alpha}
$$

gives rise to unstable solution of equations of motion and to development of vacuum condensate that compensates vacuum energy. In contrast to the energy-momentum tensor of the considered above scalar field, the energy-momentum tensor of $\Phi_{\mu \nu}$ is of vacuum form, i.e. proportional to $g_{\mu \nu}$. Such a theory possesses a symmetry with respect to transformation $\Phi_{\mu \nu} \rightarrow \Phi_{\mu \nu}+C g_{\mu \nu}$. This symmetry prevents from quantum generation of mass of $\Phi_{\mu \nu}$ and may be helpful in some other respects. Still in the simplest versions of the model the gravitational coupling constant evolves with time in the same way as in the scalar field case [29. Presumably it is related to the breaking of Lorents invariance by the condensate. The model permits a generalization such that the vacuum field $\Phi_{\mu \nu}$ is proportional to the metric tensor, $g_{\mu \nu}$, so that the condensate is Lorents invariant. However in any case the cosmology is far from being realistic. Thus, though the compensation mechanism shows some nice features, no workable model giving realistic cosmology is found at the present day.

Stimulated by the indications that the universe may expand with acceleration, i.e. that $\rho_{\text {vac }}>0$, a new constant parameter $w$, was introduced into the standard set of cosmological parameters [30]. This parameter characterizes the equation of state of the cosmological matter:

$$
p=w \rho
$$

In the standard cosmology it is assumed that the universe is now dominated by non-relativistic matter, so that $w=0$. At an earlier stage relativistic matter was dominating and $w=1 / 3$. In the case of dominance of vacuum energy $w=-1$. Two more examples giving a negative $w$ are the system of non-interacting cosmic strings with $w=-1 / 3$ and also non-interacting domain walls with $w=-2 / 3$. Since the source of gravity in General Relativity (in isotropic case) is $(\rho+3 p)$, the universe would expand with acceleration (anti-gravity) if $w<-1 / 3$.

In particular a model with a massless or extremely light scalar field was discussed that could give a negative $w$. Such field received the name "quintessence". For a homogeneous scalar field $\phi(t)$ with a self-interaction potential $U(\phi)$ the parameter $w$ is given by:

$$
w=-\frac{2 U(\phi)-\dot{\phi}^{2}}{2 U(\phi)+\dot{\phi}^{2}}
$$

If the potential energy is larger than the kinetic one, $w$ would be negative. However in this model $w$ may be considered as a constant only approximately. A fundamental theory that requests an existence of such a field is missing so such model can be considered as a poor man phenomenologv describing an accelerated expansion more oeneral than iust that given by 
vacuum energy. A raison d'être for such a field could be the adjustment mechanism discussed above, that predicts an existence of non-compensated vacuum energy with an unusual equation of state. Simultaneously, as mentioned above, the adjustment mechanism may explain the puzzling fact that the contribution of quintessence into $\Omega$ is close to 1 .

One can see from eq. (12) that the lower limit for $w$ is $w>-1$ and this is quite generic for any normal matter. However in ref. [31] even a possibility of $w<-1$ was discussed with an appropriate name "cosmic phantom". Such really striking equation of state could be realized in models with higher rank tensor fields but it gives rise to a very unusual cosmological singularity (see discussion in ref. [28]).

\section{Neutrino.}

Neutrino as a possible candidate for dark matter has the following two advantages. First, it is the only one that is definitely known to exist. Second, neutrino should have a non-zero mass. There are recent indications [32] that at least one neutrino species has a mass about $0.07 \mathrm{eV}$. However the second advantage is simultaneously a disadvantage, because the neutrino mass is normally too small for an appropriate description of the large scale structure of the universe. If cosmic background neutrinos of the $a$-th flavor have the standard cosmological abundance, $n_{\nu_{a}}=3 n_{\gamma} / 11 \approx 112 / \mathrm{cm}^{3}$, then their mass is is restricted by the Gerstein-Zeldovich [33] bound:

$$
\sum_{a} m_{\nu_{a}}<94 \mathrm{eV} \Omega h_{100}^{2}
$$

Such light neutrinos decoupled from cosmic plasma while they were relativistic and they erased all structures by free streaming at the scales below

$$
M_{\text {struc }} \sim \frac{m_{P l}^{3}}{m_{\nu}^{2}} \approx 10^{15} M_{\odot}\left(\frac{10 \mathrm{eV}}{m_{\nu}}\right)^{2}
$$

This is typical example of a hot dark matter. (A more accurate estimate gives somewhat smaller $M_{\text {struc. }}$ )

On the other hand the Tremain-Gunn bound [34] demands that neutrino mass is bounded from below:

$$
m_{\nu}>50-100 \mathrm{eV}
$$

This bound is a striking example of quantum effects on galactic scale: Fermi exclusion principle forbids too many neutrinos to accumulate in galactic halo, hence to carry all observed mass they should be sufficiently heavy.

The mismatch between the bounds (13) and (15) does not allow the standard neutrinos to constitute all dark matter in the universe. However, if neutrinos possess a new interaction somewhat stronger than the usual electroweak one, their cosmological number density would be smaller and the limit (13) would be less restrictive. Another possibility is that there are the so called sterile neutrinos that may be mirror or shadow neutrinos (see sec. 8) with the mass in $\mathrm{keV}$ range thus providing warm dark matter [35].

Some time ago a very heavy neutrino with the mass in $\mathrm{GeV}$ range was considered as a feasible candidate for cold dark matter. However the combined LEP result 36 of precise measuring of $Z^{0}$ width permits only $N_{\nu}=2.993 \pm 0.011$ for all neutral fermions with the normal weak coupling to $Z^{0}$ and mass below $m_{Z} / 2 \approx 45 \mathrm{GeV}$. So if heavy neutrinos, $\nu_{h}$, of the 
fourth generation exist their mass must be higher than $45 \mathrm{GeV}$. Most probably such particles should be unstable but if the corresponding leptonic charge is conserved or almost conserved and the charged companion of the heavy neutrino is heavier than $\nu_{h}$ they would be stable or very long lived.

The contribution of $\nu_{h}$ into cosmological energy density is determined by the cross-section of $\nu_{h} \bar{\nu}_{h}$-annihilation and has a rather peculiar behavior as a function on the $\nu_{h}$ mass. The corresponding $\Omega$ is presented in fig. 3. In the region of very small masses the ratio of number

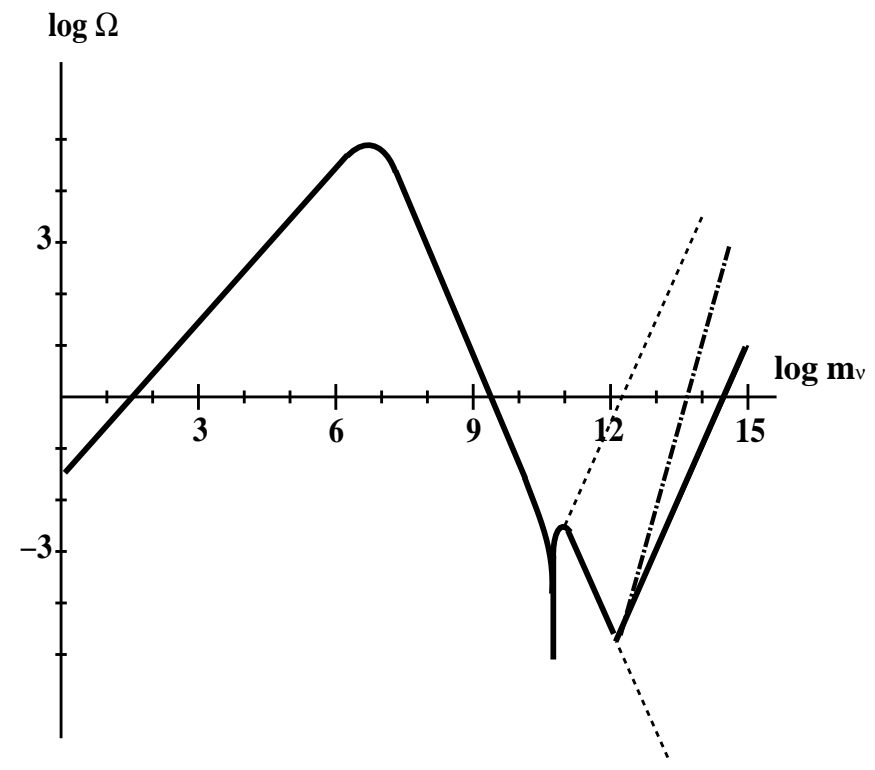

Figure 3: Contribution to cosmological parameter $\Omega$ from a heavy stable neutrino as as function of its mass.

densities $n_{\nu_{h}} / n_{\gamma}$ does not depend upon the neutrino mass and $\rho_{\nu_{h}}$ linearly rises with mass. This gives the bound (13). For larger masses $\sigma_{\text {ann }} \sim m_{\nu_{h}}^{2}$ and $\rho_{\nu_{h}} \sim 1 / m_{\nu_{h}}^{2}$. This formally opens a window for $m_{\nu_{h}}$ above $2.5 \mathrm{GeV}$ [37, 38]. A very deep minimum in $\rho_{\nu_{h}}$ near $m_{\nu_{h}}=m_{Z} / 2$ is related to the resonance enhanced cross-section around $Z$-pole. Above $Z$-pole the cross-section of $\bar{\nu}_{h} \nu_{h}$-annihilation into light fermions goes down with mass as $\alpha^{2} / m_{\nu_{h}}^{2}$ (as in any normal weakly coupled gauge theory). The corresponding rise in $\rho_{\nu_{h}}$ is shown by a dashed line. This would give the limit $m_{\nu_{h}}<3-5 \mathrm{TeV}$ [39, 40]. However for $m_{\nu_{h}}>m_{W}$ the contribution of the channel $\bar{\nu}_{h} \nu_{h} \rightarrow W^{+} W^{-}$leads to the rise of the cross-section with the increasing mass as $\sigma_{\text {ann }} \sim \alpha^{2} m_{\nu_{h}}^{2} / m_{W}^{4}$ [41. This would permit to keep $\rho_{\nu_{h}}$ well below $\rho_{c}$ for all masses above $2.5 \mathrm{GeV}$. The behavior of $\rho_{\nu_{h}}$ with this effect of rising cross-section included, is shown by the solid line till $m_{\nu_{h}}=1.5 \mathrm{TeV}$. Above that it is continued as a dashed line. This rise with mass would break unitarity limit for partial wave amplitude when $m_{\nu_{h}}$ reaches $1.5 \mathrm{TeV}$ (or $3 \mathrm{TeV}$ for Majorana neutrino) [42, 43]. If one takes the maximum value of the S-wave cross-section permitted by unitarity, which scales as $1 / m_{\nu_{h}}^{2}$, this would give rise to $\rho_{\nu_{h}} \sim m_{\nu_{h}}^{2}$ and it crosses $\rho_{c}$ at $m_{\nu_{h}} \approx 200 \mathrm{TeV}$. This behavior is continued by the solid line above $1.5 \mathrm{TeV}$. However for $m_{\nu_{h}} \geq$ a few $\mathrm{TeV}$ the Yukawa coupling of $\nu_{h}$ to the Higgs field becomes strong and no reliable calculations of the annihilation cross-section has been done in this limit. Presumably the crosssection is much smaller than perturbative result and the cosmological bound for $m_{\nu_{h}}$ is close to several $\mathrm{TeV}$. This possible, though not certain, behavior is presented by the dashed-dotted line. 


\section{Super-heavy relics.}

Super-heavy quasi-stable particles with the mass around $10^{13} \mathrm{GeV}$ were introduced recently in refs. 44, 45, 46] to avoid the GKZ-cutoff [47] for ultra-high energy cosmic rays. These particles could have produced at the end of inflation by coherent oscillations of the inflaton field (for possible mechanisms of production see e.g. ref. 44, 49]). Some cosmological and astrophysical constraints on superheavy quasistable relics were discussed earlier in refs. [50]. Such particles may have an interesting impact on structure formation and are discussed in more details in this conference by H. Ziaeepour. However their meta-stability is rather mysterious. As was argued many years ago by Zel'dovich [51], even if baryonic charge is microscopically conserved, proton may decay through formation and subsequent evaporation of a virtual black hole. In accordance with his estimate the proton should decay with the life-time:

$$
\tau_{p} \approx \frac{1}{m_{p}}\left(\frac{m_{P l}}{m_{p}}\right)^{4} \approx 10^{45} \text { years }
$$

This estimate can be obtained as follows. The cross-section of the gravitational capture of a particle by the black hole with mass $M$ is equal to its Schwarzschild radius squared,

$$
\sigma_{\text {grav }} \approx r_{g}^{2}=\frac{M^{2}}{m_{p l}^{4}}
$$

where $m_{P l}=1.2 \times 10^{19} \mathrm{GeV}$ is the Planck mass. For the virtual black hole state, which is formed in the process of the gravitational decay of a particle with mass $m$, the mass of black hole is around the initial particle mass, $M \sim m$. Assuming that all other dimensional parameters are also close to $m$ we obtain the result (16).

We can obtain another (and different) estimate for the proton life-time using the following arguments. The amplitude of the collapse of a particle $x$ with mass $m_{x}$ into black hole with the same mass is proportional to the overlap integral:

$$
A_{\text {coll }} \sim \int d^{3} r \psi_{x} \Psi_{B H}
$$

where $\psi_{x}$ and $\Psi_{B H}$ are the wave functions of the particle and black hole. The particle wave function is localized on its Compton wave length, $l_{C}=1 / m_{x}$, while the black hole wave function is localized at $r_{g}=m_{x} / m_{P l}^{2}$. Evaluating this integral and assuming again that all other dimensional parameters are close to $m_{x}$ we obtain

$$
\tau_{x} \sim \frac{1}{m_{x}}\left(\frac{m_{P l}}{m_{x}}\right)^{n}=10^{-24+19 n}\left(\frac{\mathrm{GeV}}{m_{x}}\right)^{n+1} \mathrm{sec}
$$

where the power $n$ is equal to 6 , in contrast to $n=4$ in eq. (16).

Later on this conjecture was supported by the arguments that quantum gravity effects should break all global symmetries [52], in particular due to formation of baby universes [53]. Effective Lagrangian which describes these phenomena contains different terms with different powers of Planck mass, $m_{P l}^{4-d}$, where $d$ is called the dimension of the corresponding operator. In the examples considered above $d$ was equal to 6 and 7. The very dangerous terms are those with $d=5$. They would lead to the proton decay with life-time $\tau_{p} \sim 10^{13}$ sec, which is well below existing limits. This makes one to believe that the operator with $d=5$ do not appear in effective Lagrangian. Note, that the simple estimates presented above give $d>5$. If the 
particle decay is generated by the operator with dimension $d$ then its life-time is given by the expression (19) with $n=2(d-4)$. Thus if we demand that the particle $x$ lives longer that the universe age, $t_{U} \approx 10^{18} \mathrm{sec}$, then its mass should be bounded from above:

$$
m_{x}<10^{(19 n-42) /(n+1)} \mathrm{GeV}
$$

If the Zeldovich estimate 51$]$ is correct then $m_{x}<10^{7} \mathrm{GeV}$. If we use the estimate of the present paper which gives $n=6$, then $m_{x}<10^{10.3} \mathrm{GeV}$. The condition that these particles are heavier than $10^{13} \mathrm{GeV}$, so that their decays explain the origin of ultra-energetic cosmic rays, demands a rather high value $n>9$. The dimension of the corresponding operators should be bigger than 8.5 .

Of course the arguments presented above are not rigorous but still the gravitational decay mechanism looks very plausible. This mechanism is quite generic and does not depend upon the particle properties but only on their masses. This is related to the universality of gravitational interactions. Of course the presented estimates are rather naive and the unknown non-perturbative dynamics of quantum gravity may significantly change these results. It is possible in particular that the formation of a virtual black hole proceeds as some kind of tunneling process. In this case the decay probability might be suppressed as $\exp \left(-c m_{P l} / m_{x}\right)$ (where $c$ is a constant) and the discussed here mechanism would be ineffective.

A possible way to avoid the gravitational decay is to assume that the particle in question is the lightest in the family of particles possessing a conserved charge, which is associated with a local (gauge) symmetry (similar to electromagnetic $U(1)$ ). However it would imply that this particle is absolutely stable. To avoid that one would have to assume that the corresponding gauge symmetry is slightly broken in such a way that the gauge boson(s) acquires a tiny but non-zero mass. It is well known that black holes may have only hairs which are related to the long range forces which in turn are associated with zero mass of the particles which transmit interactions. For example Coulomb field of electrically charged black hole is maintained outside the gravitational radius only because photon is strictly massless. In the case that photon has a non-zero mass, a black hole would not have electric hairs even if electric charge is strictly conserved. A limiting transition from the case of strictly massless photon to that with a small mass is achieved by a long time of disappearance of the hairs. This time should be inversely proportional to the mass. So in principle there may exist very heavy and very long lived particles if they possess a conserved charge but the corresponding gauge symmetry is a little broken so that the gauge boson acquire a tiny mass. The charge may remain strictly conserved but the particle would be unstable in the same way as proton becomes unstable due to the collapse into black hole, despite conservation of baryonic charge in particle interactions without gravity. A possible way to realize such a model is to assume a nonminimal and gauge non-invariant coupling of gauge bosons to gravity, for example in the form $A_{\mu}^{2} R$ or $A_{\mu} A_{\nu} R^{\mu \nu}$, where $R$ is the curvature scalar and $R^{\mu \nu}$ is the Ricci tensor.

Barring this a highly speculative possibility we have to conclude that either the explanation of the highest energy cosmic rays by decays of ultra-heavy long-lived particles is impossible, because such particles should undergo fast $\left(\tau_{x}<t_{U}\right)$ decay or that the gravitational breaking of global symmetries is not as strong as we assumed above.

\section{$7 \quad$ Lightest supersymmetric particle (LSP).}

Low energy supersymmetry has at least two attractive features for a solution of dark matter problem. First the theorv predicts an existence of new stable particles that could constitute 
cosmological dark matter. Second, with a natural scale of supersymmetry breaking around 1 $\mathrm{TeV}$, the theory predicts that LSP would give $\Omega_{L S P} \approx 1$ without any fine tuning. The third feature, that makes this hypothesis especially attractive for experimentalists, is that for a large range of parameters of supersymmetric models these new stable particles are within the reach of of sensitivity of different existing and planned methods of their search. This subject was recently reviewed in great detail in ref. [54, 55], so I will be very brief here.

There are several possible candidates for the role of the dominating supersymmetric matter in the universe: neutralino (a mixture of gauginos, $\tilde{\gamma}+\tilde{Z}$, and higgsinos, $\tilde{h}_{1}+\tilde{h}_{2}$ ); sneutrino (a heavy supersymmetric partner of neutrino); gravitino (the supersymmetric partner of graviton, with spin 3/2); axino (the partner of axion), messenger fields related to a hidden sector of the theory, ... . Such particles (at least some of them) can be searched for directly by a registration in low background detectors $(\mathrm{Ge}, \mathrm{NaI}, \mathrm{Xe}, \ldots)$ through the reaction: $N+$ Nuclei $\rightarrow$ recoil. There are also indirect methods based on search for the products of their annihilation in the Earth or in the Sun, producing high energy muons. At the present day only upper limits on the annihilation cross-section are established, though there are indications on annual modulation effect [56] that may be a signature of dark matter.

A very interesting feature of neutralino annihilation in the galactic halo is a production of antimatter: not only anti-protons [57] but also a noticeable fraction of anti-deuterium may be created. According to calculations of ref. [58] the flux of $\bar{D}$ at low energy, below $1 \mathrm{GeV}$, would be much larger than the flux of the secondary $\bar{D}$, produced by the normal cosmic ray collisions. The AMS mission could either register anti-deuterium from neutralino annihilation or exclude a significant fraction in the parameter space of the low energy SUSY models. There are also promising ways to register neutralino annihilation through observation of energetic positrons or gamma rays (see ref. [54 for the details).

A low energy supersymmetric extension of the minimal standard model is very natural from particle physics point of view. It supplies possibly the best candidate for the dark matter particles. In most versions of the model these particles would form weakly interacting cold dark matter, though in some cases warm dark matter is also possible. There is a very high experimental activity in search of supersymmetric particles and hopefully at the beginning of the next millennium they will be discovered or, if the nature is not favorable, a large part of the parameter space will be excluded but the mystery of dark matter will still remain.

\section{Mirror/shadow world}

The idea that our world is doubled and there exists a similar or exactly the same world coupled to ours only by gravity, was suggested long ago [59] in connection with conservation of parity, P, or combined parity, CP. Subsequently it was developed and elaborated in several papers [60]. Its popularity greatly increased after it was found that superstring theories have $G \times G$ internal symmetry group and the two identical worlds, corresponding to two groups, communicate only through gravity [61. The considered models, however, were not confined to this simplest option. In addition to gravity a new super-weak (but stronger than gravity) interaction was introduced between our and mirror particles. Moreover, a different patterns of symmetry breaking in these two worlds were considered, so that physics in our and in the mirror, or in this case better to say in the shadow world, became quite different.

At first sight the existence of a whole new world with the same or similar particle content would strongly distort successful predictions of the standard big bang nucleosynthesis (BBN) theory. The latter permits not more than one additional light fermionic species in the cosmo- 
logical plasma at $T \sim 1 \mathrm{MeV}$ (see e.g. 12]). The completely symmetric mirror world would give slightly more than 7. However, as was argued in ref. 62 the temperature of the mirror matter after inflation could be smaller than the temperature of the usual matter and thus the energy density of mirror matter during nucleosynthesis could be safely suppressed. Concrete mechanisms that could create a colder mirror world if the symmetry between the worlds was broken, were considered e.g. in refs [35, 63]. Another possible way to escape a conflict with $\mathrm{BBN}$ by the generation of lepton asymmetry through neutrino oscillations was discussed in ref. 64.

A new burst of interest to mirror/shadow matter arose after MACHO collaboration announced that the mass of the micro-lenses, they observed, is close to the solar mass (see sec. 2). A natural idea that these objects may be built from mirror matter, immediately attracted a strong attention [35, 63, 65, 66, 67]. In the case of exact symmetry between the worlds the properties of the stellar objects would be the same but the process of structure formation could be quite different by the following two reasons. First, since the the mirror matter is colder than the usual one, the mirror hydrogen recombination would be considerably earlier and the structures might start forming earlier too. Second, baryon asymmetry in the mirror world might be different from ours and it would have an important impact on primordial chemical content of the universe and galactic and stellar formation [68]. The cosmological mass fraction of mirror baryons is unknown but most probably they do not constitute all dark matter in the universe. There is one peculiar feature of this matter that it is strongly interacting and can easily loose energy through emission of mirror photons. Structure formation with this kind of dark matter would be very much different from the normal scenario with dissipationless cold dark matter. The cooling mechanisms, that are very essential for structure formation, could be either stronger or weaker. In particular, in the world with a very large fraction of mirror ${ }^{4} \mathrm{He}$ molecular cooling would be considerably less efficient.

There would be even more difference between cosmology and astrophysics of our and mirror world if the mirror symmetry is broken [35, 63]. There could be the case that there are no stable nuclei in the mirror world and thus there could not exist mirror stars with thermonuclear active core. If the mirror electrons are heavier than the usual ones, the mirror hydrogen binding energy would be larger and this would be another reason for earlier recombination. To study the history of stellar formation and evolution in such distorted world would be a very interesting exercise that could reveal essential features of the underlying physics. Except for a different astrophysics and new stellar size invisible bodies, mirror world could provide sterile neutrinos that might explain the observed neutrino anomalies though the oscillations between our neutrinos and sterile ones. In particular, among these sterile neutrinos there could be rather heavy ones with the mass in keV range that might be excellent candidates for warm dark matter.

\section{$9 \quad$ Miscellanea}

Because of lack of space and time I could not discuss many other interesting forms of dark matter. One of the favorites, axion, is discussed at this conference by Yu. Gnedin. Topological and non-topological solitons may be also quite interesting options. Though the measurements of the angular fluctuations of CMB seemingly exclude cosmic strings as dominant part of cosmological dark matter, they still may give some contribution to the total mass of the universe. Non-topological solitons, $Q$-balls, recently attracted a renewed attention [69, 70]. Primordial black holes with the log-normal mass spectrum [71] still remain an interesting possibility. There are some even more exotic candidates that are discussed in the literature; among them are 
such objects as superstrings giving super-heavy dark matter [72], domain walls with "antigravitating" equation of state, $p=-(2 / 3) \rho$ [73], or even liquid or solid dark matter [74].

Unstable dark matter remains attractive, and though it was proposed at the beginning of 80 [75], the main burst of activity was in the 90th [76]. The basic idea of introducing unstable but long-lived particles into consideration was to increase the horizon length at the time of equality between matter and radiation and to increase by that the power at large scales. Recently this idea was revived in another attempt to save a model of structure formation with pure cold dark matter [77]. The model looks quite natural from particle physics point of view if there exists a light scalar boson, familon or majoron so that a heavier neutrino, that may violate Gerstein-Zeldovich bound, could decay into this boson and lighter neutrino. It is also possible that a massive scalar boson decays into two light neutrinos. A very interesting scenario in the former case is that the scalar bosons are massive and their spectrum is two component: energetic bosons coming from the decay and non-relativistic ones formed during phase transition similar to axions. In this case the same particle may form both cold and hot (or warm) dark matter. A slightly different mechanism was proposed in ref. [78] in the frameworks of string cosmology. It was argued there that weakly interacting non-thermal relics may be produced in the course of dilaton driven inflation with the double peak spectrum that could simultaneously give cold and hot dark matter.

A very interesting form of dark matter is a self-interacting one. One possible example of the latter is given by mirror or shadow world discussed above. A few more models of selfinteracting dark matter with particles belonging to our world were considered in the literature; they were either light bosons [79], e.g. majorons or familons, or neutrinos with an anomalous self-interaction 80]. Observational evidence in favor of self-interacting dark matter was recently analyzed in ref. 81

\section{Conclusion}

As we have seen, a set of independent arguments unambiguously proves that the main part of matter in the universe is not visible and, moreover, this invisible matter is not the matter that consists of known elementary particles as e.g. protons or neutrons, or neutrinos. Existence of this unknown form of matter is a strong evidence in favor of new physics beyond the minimal standard $S U(3) \times S U(2) \times U(1)$-model $(\mathrm{MSM})$. Possibly a low energy supersymmetric extension of MSM solves the mystery of dark matter with lightest supersymmetric particle (LSP) that quite probably could be stable. However astronomical data indicate that one form of dark matter is not enough and except for cold dark matter, that might be provided by LSP, there is a very strong quest for hot and/or warm dark matter. Moreover detailed description of rotation curves at small distances indicates that dark matter may be dissipative. Quite possibly there is one more ingredient of dark matter, related to vacuum energy, that makes the situation even more mysterious.

Even if there is only one form of dark matter, the cosmic conspiracy, namely the close values of $\Omega_{\text {baryon }}$ and $\Omega_{D M}$, is quite puzzling. It demands quite a strong fine-tuning in the fundamental particle theory and at the present day no reasonable understanding of the phenomenon exists. The problem of cosmic conspiracy becomes tremendously deeper if there are several $(>2)$ forms of invisible matter with the similar contributions to $\Omega$.

An answer to an often asked question, what is the best bet for the dark matter particles, reflects not so much our knowledge of the subject but a personal attitude of the respondent. Seemingly most votes would be given to LSP and possibly the next one is the axion. An 
advantage of these two is that both were not invented ad hoc but were predicted by particle theory independently of cosmology. By similar arguments mirror or shadow matter is also in a good shape. However other candidates based on more complicated models may have better chances just because their properties are chosen in accordance with cosmological demands.

10 years ago in one of "Rencontre de Moriond" meeting P. Peebles in his summary talk arranged a public opinion pool, how many dark matter candidates would survive to the end of the century. The stakes were up to double digit numbers. I have to admit that I voted for one dark matter candidate, the only real one that "would be surely known". It was extremely over-optimistic point of view and today we have even more possible candidates than 10 years ago (neither old ones is removed from the list and quite a few new ones came into being) and still do not know what is/are the correct one(s).

Acknowledgments The work of A.D. was supported by Danmarks Grundforskningsfond through its funding of the Theoretical Astrophysical Center.

\section{References}

[1] J.H. Oort, Bull. Astron. Inst. Netherlands, 6, 2491932.

[2] F. Zwicky, Helv. Phys. Acta, 6, 110, 1933.

[3] J. Einasto, A. Kaasik and E. Saar, Nature, 250, 309, 1974.

[4] J.P. 0striker, P.J.E. Peebles and A. Yahil, Astrophys. J. 193, L1, 1974.

[5] G. Raffelt, Proceedings of the 1997 European School of High-Energy Physics, Menstrup near Naestved, Denmark; hep-ph/9712538.

[6] P. Salucci, M. Persic, Proceedings of the Sesto DM1996 Conference Eds M. Persic \& P. Salucci. ASP Proceedings Conference Series 1997; astro-ph/9703027.

[7] M. Persic, P. Salucci, Astrophys. J. Suppl. 99, 501, 1995.

[8] S. Sakai, J.R. Mould, S.M.G. Hughes, et al, astro-ph/9909269;

T. Richtler, G. Drenkhahn, astro-ph/9909117, to be published in: Kundt W., van de Bruck

C. (Eds.) Cosmology and Astrophysics: A collection of critical thoughts. Lecture Notes in Physics, Springer;

L.V.E. Koopmans, C. D. Fassnacht, astro-ph/9907258, Astrophys. J. (to be published).

[9] W.L. Freedman, astro-ph/9909076, to be published in the David Schramm Memorial Volume of Physics Reports.

[10] M. Persic, P. Salucci, MNRAS, 258, 14, 1992.

[11] G. Gamow, Phys. Rev. 70, 527, 1946 ;

R.A. Alpher, H. Bethe, G. Gamow, Phys. Rev. 73, 803, 1948.

[12] K.A. Olive, G. Steigman, T.P. Walker, to be published in a special memorial volume of Physics Reports in honor of David Schramm; astro-ph/9905320. 
[13] A.D. Dolgov and B.E.J. Pagel, TAC-1997-038, astro-ph/9711202. To be published in New Astronomy (dedicated to memory of David Schramm);

A.D. Dolgov, TAC-1999-001; astro-ph/9901318. "Particle Physics and the Early Universe (COSMO-98)", p.259; ed. by David O. Caldwell, American Institute of Physics.

[14] Wendy L. Freedman, Invited Review given at the Nobel Symposium, "Particle Physics and the Universe, Haga Slott, Sweden, August 1988, to be published by World Scientific Press; astro-ph/9905222.

[15] S. Perlmutter, G. Aldering, G. Goldhaber, et al, to be published in Astrophys. J.; astroph/9812133.

[16] Adam G. Riess, Alexei V. Filippenko, Weidong Li, Brian P. Schmidt, to be published in Astronomical Journal; astro-ph/9907038

[17] G. Gamow, "My World Line" Viking, New York, 1970.

[18] M. Fukugita, C. J. Hogan, P. J. E. Peebles, Astrophys. J. 503, 518, 1998.

[19] K.A. Olive, D.N. Schramm, D. Thomas, and T.P. Walker, Phys. Lett. B65, 239, 1991.

[20] H.-O. Kang and G. Steigman, Nucl. Phys. B, 372, 494, 1992.

[21] G. Rocha, astro-ph/9907312, Invited review to appear in the proceedings of the 'Early Universe and Dark Matter Conference', DARK98, Heidelberg, 1998.

[22] B. Paczynski, Astrophys. J. 301, 503, 1992.

[23] W. Sutherland, astro-ph/9811185; Rev. Mod. Phys. 'Colloquium' article, 71, 421, 1999.

[24] EROS Collaboration, astro-ph/9812173; A\&A Letters (to be published); F. Derue, C. Afonso, C. Alard, J-N. Albert, et al, (The EROS collaboration) astroph/9903209, A\&A (to be published).

[25] S. Weinberg, Rev. Mod. Phys. 61, 1, 1989.

[26] A.D. Dolgov, A lecture presented at the 4th Colloque Cosmologie, Paris, June, 1997. "Fourth Paris Cosmology Colloquium", World Scientific, 1998, Singapore, 161-175. Ed. H.J. De Vega and N. Sanchez.

[27] A.D. Dolgov, In Proc. Very early Universe. Nuffield Workshop, Cambridge, 1982. Ed. G.W. Gibbons, S.W. Hawking, and S. Siklos; 449-458. Cambridge Univ. Press, 1983.

[28] A.D. Dolgov, Phys.Rev. D55, 5881, 1997.

[29] V. A. Rubakov, P. G. Tinyakov, hep-ph/9906239.

[30] R. R. Caldwell, R. Dave, P.J. Steinhardt, Phys.Rev.Lett. 80, 1582, 1998;

G. Huey, L. Wang, R. Dave, R. R. Caldwell, P.J. Steinhardt, Phys.Rev. D59, 063005, 1999.

[31] R. R. Caldwell, astro-ph/9908168.

[32] Y. Fukuda et al, Phys. Rev. Lett. 81, 1562, 1998. 
[33] S.S. Gerstein and Ya.B. Zeldovich, Pis'ma ZhETF, 4, 174, 1966; (English translation: JETP Letters, 4, 120, 1966).

[34] S. Tremain, J. Gunn, Phys. Rev. Lett. 42, 407, 1979.

[35] Z.G. Berezhiani, R.N. Mohapatra, Phys. Rev. D52, 6607, 1995;

Z.G. Berezhiani, A.D. Dolgov, R.N. Mohapatra, Phys. Lett. B375, 1996, 26.

[36] Particle Data Group, European Physical Journal C, 3, 1, 1998.

[37] M.I. Vysotsky, A.D. Dolgov and Ya.B. Zeldovich, Pis'ma ZhETF, 26, 200, 1977.

[38] B.W. Lee and S. Weinberg, Phys. Rev. Lett. 39, 165, 1977.

[39] A.D.Dolgov, Ya.B.Zeldovich, Rev. Mod. Phys. 53, 11981.

[40] K.A. Olive, M.S. Turner, Phys. Rev. D25, 213, 1982.

[41] K. Enqvist, K. Kainulainen, J. Maalampi, Nucl.Phys. B317, 647, 1989.

[42] K. Griest and M. Kamionkowski, Phys. Rev. Lett. 64, 615, 1990.

[43] K. Enqvist, K. Kainulainen, Phys. Lett. B264, 367, 1991.

[44] V. Berezinsky, M. Kachelrieß, A. Vilenkin, Phys. Rev. Lett. 79, 4302, 1997.

[45] V.A. Kuzmin, V.A. Rubakov, Phys.Atom.Nucl. 61, 1028, 1998; Yad.Fiz. 61, 1122, 1998.

[46] M. Birkel, S. Sarkar, Astropart. Phys. 9, 297, 1998.

[47] K. Greisen, Phys. Rev. Lett. 16, 748, 1966;

G. T. Zatsepin and V. A. Kuzmin, Pis'ma Zh. Eksp. Teor. Fiz. 4, 14, 1996 [English translation: JETP. Lett. 4, 78, 1966.

[48] D.J H. Chung, E. W. Kolb, A. Riotto, Phys.Rev.Lett. 81, 4048, 1998.

[49] V.A. Kuzmin, I.I. Tkachev, Phys.Rev. D59, 123006, 1999.

[50] J. Ellis, G.B. Gelmini, J.L. Lopez, D.V. Nanopoulos, S. Sarkar Nucl. Phys. B373, 399, 1992;

P. Gondolo, G. Gelmini, S. Sarkar, Nucl.Phys. B392, 111, 1993.

[51] Ya.B. Zeldovich, JETP Letters, 24, 29, 1976.

[52] R. Barbieri, J. Ellis, M.K. Gaillard, Phys. Lett. 90B, 249 1980;

T. Banks, Physicalia, 12, 19, 1990;

R. Holman, S.D.H. Hsu, T. Kephart, E. Kolb, R. Watkins, L. Widrow, Phys. Lett. B282, 13, 1992;

M. Kamionkowski, J. March-Russel, Phys. Lett. B282, 137, 1992;

E.Kh. Akhmedov, Z.G. Berezhiani, G. Senjanovic, Phys. Rev. Lett., 69, 3013, 1992;

S. Barr and D. Seckel, Phys. Rev. D46, 539, 1992;

D. Grasso, M. Lusignoli, M. Roncadelli, Phys. Lett. B288, 140, 1992;

S. Ghigna, M. Lusignoli, M. Roncadelli, Phys. Lett. B288, 278, 1992;

E.Kh Akhmedov Z.G. Berezhiani. R.N Mohanatra G. Senianovic. Phys Lett. B299. 
3013, 1993;

K.S. Babu, S.M. Barr, Phys. Lett. B300, 376, 1993;

I.Z. Rothstein, K.S. Babu, D. Seckel Nucl. Phys. B403, 725, 1993.

[53] S. Giddings and A. Strominger, Nucl. Phys. B307, 854, 1988;

S. Coleman, Nucl. Phys. B310, 643, 1988.

[54] A. Bottino, N. Fornengo, hep-ph/9904469, Lectures given at the Fifth School on NonAccelerator Particle Astrophysics (Abdus Salam International Centre for Theoretical Physics, Trieste, June-July 1998), to appear in the School Proceedings (Eds. R.A. Carrigan, Jr., G. Giacomelli and N. Paver).

[55] A. Masiero, F. Rosati, hep-ph/9905476, 8th International Workshop on Neutrino Telescopes, 23-26 Feb. 1999, Venice, Italy, to be published in the Proceedings.

[56] R. Bernabei, et al, Phys. Lett. B424, 195, 1998;

P. Belli, R. Bernabei, A. Bottino, et al, hep-ph/9903501.

[57] P. Chardonnet, G. Mignola, P. Salati, R. Taillet, Phys. Lett. B384, 161 1996;

T. Mitsui, K. Maki, S. Orito, Phys. Lett. B389, 169, 1996;

J.D. Wells, A. Moiseev, J.F. Ormes, Astrophys.J. 518, 570, 1999;

L. Bergstrom, J. Edsjo, P. Ullio, astro-ph/9902012, astro-ph/9906034.

[58] F. Donato, N. Fornengo, P. Salati, hep-ph/9904481.

[59] T.D. Lee, C.N. Yang, Phys.Rev. 104, 254, 1956;

A. Salam, Nuovo Cimento, 5, 299, 1957;

K. Nishijima, M. Saffouri, Phys. Rev. Lett. 14, 205, 1965;

I. Yu. Kobzarev, L.B. Okun, I.Ya. Pomeranchuk, Sov. J. Nucl. Phys., 3, 837, 1966.

[60] L.B. Okun, ZHETF, 79, 694, 1980;

S.I. Blinnikov, M.Yu. Khlopov, Sov. Astron. J. 60, 632, 1983;

B. Holdom, Phys. Lett. B166, 196, 1985;

S.L. Glashow, Phys. Lett. B167, 35, 1986;

E.D. Carlson, S.L. Glashow, Phys. Lett. B193, 168, 1987;

M. Khlopov et al, Sov. Astron. J. 68, 42, 1991;

R. Foot, H. Lew, R. Volkas, Phys. Lett. B272, 67, 1991; Mod. Phys. Lett. A7, 2567, 1992;

R. Foot, R. R. Volkas, Phys.Rev. D52, 65951995.

[61] M. Green, J. Schwarz, E. Witten, "Superstring Theories", Cambridge University Press, 1989.

[62] E. Kolb, D. Seckel, M. Turner, Nature, 514, 415, 1985.

[63] Z. G. Berezhiani, Acta Phys.Polon. B27, 1503, 1996.

[64] R. Foot, R. R. Volkas, Astropart. Phys. 7, 283, 1997.

[65] S. I. Blinnikov, astro-ph/9801015, presented at "Baryonic Matter in the Universe and Its Spectroscopic Studies" November 22 - 24, 1996, Atami, Japan);

S. I. Blinnikov, astro-ph/9902305, presented at XXVII ITEP Winter School, Snegiri, Feb. $16-24,1999$. 
[66] R. Foot, Phys.Lett. B452, 83, 1999;

R. Foot, R. R. Volkas, hep-ph/9904336;

R. Foot, astro-ph/9908276.

[67] R.N. Mohapatra, V.L. Teplitz, astro-ph/9902085.

[68] Z. Berezhiani, D. Comelli, talk presented at COSMO-99, Trieste, September, 1999.

[69] A. Kusenko, M. Shaposhnikov, Phys.Lett. B418, 46, 1998;

A. Kusenko, hep-ph/9901353," Particle Physics and the Early Universe (COSMO-98)", ed. by David O. Caldwell, American Institute of Physics.

[70] K. Enqvist, J. McDonald, Phys.Lett. B440, 59 1998;

K. Enqvist, J. McDonald, Nucl.Phys. B538, 321, 1999.

[71] A. Dolgov, J. Silk, Phys. Rev. D47, 4244, 1993.

[72] G. Dvali, Phys.Lett. B459, 4891999.

[73] R. A. Battye, M. Bucher, D. Spergel, astro-ph/9908047.

[74] D. Eichler, Astrophys. J. 468, 75, 1996.

[75] M. Davis, M. Lecar, C. Pryor, E. Witten, Astrophys. J. 250, 423, 1981;

G. Gelmini, D. N. Schramm, J. W. F. Valle, Phys. Lett. B146, 311, 1984;

A.G. Doroshkevich, M.Yu. Khlopov, Yadernaya Fizika, 39, 869, 1984; MNRAS, 211, 277, 1984

M.S. Turner, G. Steigman, L.M. Krauss. Phys. Rev. Lett. 52, 2090, 1984;

P.Hut, S.D.M. White, Nature, 310, 637, 1984;

K.A. Olive, D. Seckel, E. Visniac, Astrophys. J. 292, 1, 1985;

J. Bardeen, J. Bond, G. Efstathiou, Astrophys. J. 321, 28, 1987;

A.G. Doroshkevich, A.A. Klypin, M.Yu. Khlopov, Astron. J. 65, 248, 1988; MNRAS, 239, 923, 1989;

[76] J. Bond, G. Efstathiou, Phys. Lett, B265, 245, 1991;

M. Davis et. al. Nature, 356, 489, 1992;

S. Dodelson, G. Gyuk, M. Turner, Phys. Rev. Lett. 72, 3754, 1994;

H. Kikuchi, E. Ma, Phys. Rev. D51, 296, 1995;

H. B. Kim and J. E. Kim, Nucl. Phys. B433, 421, 1995;

A. S. Joshipura, J. W. F. Valle, Nucl. Phys. B440, 647, 1995;

M. White, G. Gelmini, J. Silk, Phys. Rev. D51, 2669, 1995;

A.D. Dolgov, S. Pastor, J.W.F. Valle, FTUV/95-14, IFIC/95-14, asto-ph/9506011.

[77] A. Masiero, D. Montanino, M. Peloso, hep-ph/9902380, to be published in Astropart. Phys.

[78] R. Brustein, M. Hadad. Phys. Rev. Lett. 82, 3016, 1999.

[79] A. D. Dolgov, Yadernaya Fizika (Sov. J. Nucl. Phys.), 31, 1522, 1980;

E. D. Carlson, M. E. Machacek, L.J. Hall, Astrophys. J. 398, 43, 1992;

M. E. Machacek, Astrophys. J. 431, 431, 1994;

A. A. de Laix, R. J. Scherrer, and R. K. Schaffer, Astrophys. J. 464, 539, 1996;

A.D. Dolgov, S. Pastor, J.W.F. Valle, FTUV/95-14, IFIC/95-14, asto-ph/9506011. 
[80] F. Atrio-Barandela, S. Davidson, Phys.Rev. D55, 58861997.

[81] D.N. Spergel, P.J. Steinhardt, astro-ph/9909386. 